\title{
A FRUTICULTURA COMO ALTERNATIVA PARA A PRODUÇÃO FAMILIAR NO ÂMBITO DO PRONAF NOS MUNICÍPIOS DE CAMPOS DOS GOYTACAZES E SÃO FRANCISCO DO ITABAPOANA - RJ'
}

\author{
VICTOR RODRIGUES FERREIRA², PAULO MARCELO DE SOUZA³, NIRALDO JOSÉ PONCIANO ${ }^{4}$, ALMY JÚNIOR \\ CORDEIRO DE CARVALHO
}

RESUMO - O trabalho analisa a importância do cultivo de frutas como alternativa para o aumento de renda da agricultura familiar, no contexto dos financiamentos concedidos pelo PRONAF. Os resultados evidenciaram que o recurso financeiro foi a variável que mais limitou a escolha dos agricultores. Maracujá, goiaba e pinha foram identificadas como as melhores alternativas econômicas para os produtores familiares da região. A análise permitiu concluir que a concessão do crédito, pelo PRONAF, contribui para a melhor utilização dos recursos, para a obtenção de maior renda, bem como para a geração de empregos.

Termos para indexação: agricultura familiar, programação linear, PRONAF.

\section{THE HORTICULTURE AS ALTERNATIVE FOR FAMILY PRODUCTION IN THE CONTEXT OF PRONAF IN THE MUNICIPAL DISTRICTS OF CAMPOS DOS GOYTACAZES AND SÃO FRANCISCO DO ITABAPOANA - RJ}

ABSTRACT - the work analyzes the importance of the culture of fruits as alternative for increasing the income of familiar agriculture, in the context of financial granted by PRONAF. The results had evidenced that the financial resource was the variable that more limited the choice of the farmers. Passion fruit, guava and sugar apple had been identified as the best economic alternatives for the familiar farmers of the region. The analysis allowed to conclude that the concession of the credit, by the PRONAF, contributes for the best use of the resources, for the attainment of bigger income, as well as for the generation of jobs.

Index terms: family agriculture, lineal programming, PRONAF.

\section{INTRODUÇÃO}

Em estudo realizado pela FAO/INCRA(1996) foi constatada a importância da agricultura familiar, do ponto de vista estratégico, para manutenção e recuperação do emprego, para redistribuição da renda, para garantia de alimentos e para o desenvolvimento sustentável. Em 1995, dos 4,13 milhões de estabelecimentos rurais no Brasil, $85 \%$ podem ser classificados como pequenas propriedades, ocupando apenas $30 \%$ da área total do país. Estes correspondem com $38 \%$ do valor da produção no Brasil e mantêm aproximadamente 13,8 milhões de pessoas ocupadas, equivalentes a mais de $75 \%$ da mão-de-obra agropecuária (Fundação Instituto Brasileiro de Geografia e Estatística - FIBGE, 2001).

Não obstante sua importância, a agricultura familiar passa por vários problemas, como a falta de assistência técnica adequada, a falta de uma política agrícola mais eficiente e principalmente a insuficiência de recursos financeiros. A criação do PRONAF - Programa Nacional de Fortalecimento daAgricultura Familiar, em 1996, está conformando um novo campo de coalizão de atores, públicos e privados, tendo por objetivo o tratamento ideal às necessidades da agricultura familiar, inclusive à capacitação dos agricultores.

Na região Norte Fluminense, tem havido estímulos ao desenvolvimento da fruticultura, dado algumas características propícias, como solo, possibilidade de irrigação, proximidade de grandes centros consumidores, instalação de agroindústrias etc. Nesse sentido, essa atividade pode aumentar a rentabilidade dos produtores da região e ocupar, de forma racional, a mão-de-obra familiar disponível. Partindo dessa perspectiva, o trabalho teve como objetivo verificar a importância da fruticultura na viabilização da produção familiar, buscando determinar a combinação ótima de culturas, considerando as limitações de recursos desses agricultores, bem como as possibilidades de financiamento pelo PRONAF.

\section{MATERIALE MÉTODOS}

Do ponto de vista do público-alvo, o PRONAF atende especificamente os agricultores familiares, que são caracterizados a partir dos seguintes critérios: possuir $80 \%$ da renda familiar originária da atividade agropecuária; deter ou explorar estabelecimentos com área de até quatro módulos fiscais; explorar a terra na condição de proprietário, meeiro, parceiro ou arrendatário; utilizar mão-de-obra exclusivamente familiar, podendo manter até dois empregados permanentes; residir no imóvel ou em aglomerado rural ou urbano próximo e possuir uma renda anual máxima de até R\$27.500,00 (BRASIL. Ministério do Desenvolvimento Agrário, 2001). Os agricultores familiares beneficiários do PRONAF são divididos em quatro grupos distintos para fins de concessão dos financiamentos, definidos com base na renda bruta anual, bem como no tipo de mão-de-obra empregada:

Grupo A - Agricultores familiares assentados pelo Programa Nacional de Reforma agrária que não contrataram operação de investimento no limite individual permitido pelo antigo PROCERA (R\$ 7.500,00).

Grupo B - Agricultores familiares de mínima renda, que se assalariam; ou assalariados rurais com estabelecimento rural; ou famílias com estabelecimento rural exercendo atividades não agropecuárias. A mão-de-obra deve ser exclusivamente familiar. A renda bruta anual não deve pode ser superior a $\mathrm{R} \$ 1.500,00$ (excluída a aposentadoria rural).

Grupo C - Agricultores familiares descapitalizados; ou trabalhadores rurais com atividades não agropecuárias em estabelecimento rural. A mão-de-obra deve ser predominantemente familiar com possibilidade de eventual contratação de terceiros. A renda bruta anual deve estar entre $\mathrm{R} \$ 1.500,00$ e $\mathrm{R} \$ 8.000,00$, com $80 \%$ da renda provindo do estabelecimento através de exploração agropecuária ou não agropecuária.

Grupo D - Agricultores familiares ou trabalhadores rurais com maior nível de capitalização, que exerçam atividades agropecuárias ou não agropecuárias em estabelecimento rural. A mão-de-obra deve ser predominantemente familiar, podendo ter até dois empregados permanentes com possibilidade de eventual contratação de terceiros. A renda bruta anual deve estar entre $\mathrm{R} \$ 8.000,00$ e R $\$ 27.500,00$, com $80 \%$ da renda provindo do estabelecimento através de exploração agropecuária ou não agropecuária.

\footnotetext{
(Trabalho 016/2003). Recebido: 10/02/2003. Aceito para publicação: 28/08/2003.

2 Eng. Agr., M.S., Univ. Estadual do Norte Fluminense, UENF, Campos, RJ, victor@uenf.br.

3 Professor D.Sc.,Univ. Estadual do Norte Fluminense, UENF, Campos, RJ, pmsouza@uenf.br.

${ }_{5}^{4}$ Professor D.Sc., Univ. Estadual do Norte Fluminense, UENF, Campos, RJ, ponciano@uenf.br.

Professor D.Sc., Univ. Estadual do Norte Fluminense, UENF, Campos, RJ, almy@uenf.br.
} 
No que diz respeito à linha de custeio, que interessa ao presente trabalho, o Programa prevê, para os grupos B, C e D, mas não para o A, valores anuais de, respectivamente, $\mathrm{R} \$ 500,00, \mathrm{R} \$ 2.000,00$ (com 50\% adicionais para investimento em fruticultura) e $\mathrm{R} \$ 5.000,00$, com vencimento no prazo de dois anos. Os encargos anuais para os grupos C e D são de juros de $4,0 \%$ ao ano e para o grupo B, juros de $1 \%$ ao ano. Para o grupo A somente está disponível a linha de investimento do PRONAF, pois são produtores assentados pelo programa de Reforma Agrária. Assim, a quantidade de capital disponível para este grupo usada no modelo foi a quantidade de capital próprio, média descoberta nas entrevistas (BRASIL. Ministério do Desenvolvimento Agrário, 2001).

Foram realizadas entrevistas com cinco produtores de cada grupo representativo da região, visando obter dados médios sobre tamanho da propriedade, capital próprio e mão-de-obra disponível. Dessa pesquisa, foram obtidas as informações constantes da Tabela 1.

TABELA 1 - Área utilizável das propriedades, capital próprio e disponibilidade de mão-de-obra de cada grupo.

\begin{tabular}{lccc}
\hline \multicolumn{1}{c}{ Médias } & Grupo A & Grupo C & Grupo D \\
& & & \\
\hline Área (ha) & 10 & 15 & 20 \\
Capital próprio (R\$) & 5500,00 & 13120,00 & 15420,00 \\
Mão de obra (dia/homem/ano) & 690 & 690 & 690 \\
\hline FONTE: Dados da pesquisa & \multicolumn{3}{c}{}
\end{tabular}

A quantidade de capital próprio de cada grupo foi calculada a partir dos resultados dos custos anuais de cada atividade realizada pelo produtor e da quantidade de financiamento adquirida anualmente por cada produtor. Admitiu-se que a soma dos valores dos custos das atividades empregadas pelo produtor equivale aos Custos Operacionais Efetivos (COE) que, segundo Matsunaga et al. (1976), representam os gastos efetivamente realizados na condução da atividade, excluindo-se os custos correspondentes aos serviços executados pela mão-de-obra familiar e à depreciação do capital imobilizado em benfeitorias, equipamentos, animais de serviço e forrageiras não anuais. O pagamento dos Custos Operacionais Efetivos (COE) de cada produtor seria realizado pela soma dos valores de Capital Próprio (CP) mais o Valor do Financiamento Adquirido (VFA), resumido pela fórmula: $\mathrm{COE}=\mathrm{CP}+\mathrm{VFA}$. Assim, os valores de capital próprio foram calculados para cada produtor por uma simples substituição na fórmula, sendo $\mathrm{CP}=\mathrm{COE}-\mathrm{VFA}$. Com esta fórmula foram obtidas as médias de capital próprio de cada grupo do PRONAF.

$\mathrm{O}$ valor da quantidade média de mão-de-obra familiar foi obtido a partir dos dados do número de pessoas que faziam parte da família. É importante lembrar que as crianças foram computadas como meio adulto na disponibilidade da mão-de-obra familiar.

Por meio do levantamento direto com pequenos produtores e de entrevistas com técnicos da EMATER (regionais de Campos dos Goytacazes e de São Francisco do Itabapoana), buscou-se também identificar, a partir da realidade do pequeno produtor dessas regiões, o conjunto de atividades passíveis de serem cultivadas, as produtividades médias e os custos operacionais. Com essas informações, foi possível calcular a renda bruta por hectare de cada atividade, multiplicando-se a produtividade pelo preço do produto. Subtraindo-se da renda bruta os custos operacionais, obteve-se a margem bruta por hectare, referente a cada atividade. Além de frutíferas como a bananeira, o coqueiro, o abacaxizeiro, a goiabeira, o maracujazeiro, a pinheira e os citros, foram incluídas ainda culturas anuais como mandioca, cana-de-açúcar e olerícolas, que são habitualmente desenvolvidas pelos agricultores entrevistados.

O passo seguinte consistiu na aplicação da programação linear, que é uma técnica de planejamento voltada para a otimização de problemas em que se têm diversas opções de escolha sujeitas a algum tipo de restrição ou regulamentação (Prado, 1999). Segundo Puccini \& Pizzolato (1987), a solução de qualquer modelo consiste em achar $\mathrm{x}_{1}, \mathrm{x}_{2}, \ldots, \mathrm{x}_{\mathrm{n}}$ que maximize ou minimize a função linear (função objetivo), satisfazendo diversas inequações lineares (restrições), como é mostrado no modelo geral:

$\operatorname{Max.} \mathrm{Z}=\sum_{j=1}^{n} c_{j} x_{j}$

sujeito a:

$$
\begin{aligned}
& \sum_{i j=1}^{n} a_{i j} x_{j} \leq b_{i} \quad(i=1,2, \ldots, \mathrm{m}) \\
& x_{j} \geq 0 \quad(\mathrm{j}=1,2, \ldots, \mathrm{n})
\end{aligned}
$$

Considerando-se uma empresa agropecuária com $m$ recursos disponíveis para produção de $n$ produtos distintos (atividades), tem-se, para cada recurso $i$ e atividade $j$ :

$\mathrm{x}_{j}=$ total da área de produção de cada atividade $j$.

$\mathrm{c}_{j}=$ margem bruta anual de cada atividade $j$.

$\mathrm{b}_{i}^{j}=$ quantidade disponível, anualmente, do recurso $i$.

$\mathrm{a}_{i j}=$ quantidade do recurso $i$ consumida, por ha, na produção de uma unidade da atividade $j$.

A função objetivo a ser maximizada representa a margem bruta total da empresa agropecuária na combinação ótima das $n$ atividades distintas. As $m$ restrições informam que o total gasto do recurso $i$, nas $n$ atividades, tem que ser menor ou, no máximo, igual à quantidade $\mathrm{b}_{i}$ disponível daquele recurso. As restrições $\mathrm{x}_{j}=0$ indicam que a área de produção de cada cultura não pode ser negativa.

Com essa técnica, procurou-se determinar as atividades que, plantadas em cada ano, maximizassem o saldo final do agricultor ao décimo ano, respeitando-se suas restrições de capital, terra e mãode-obra disponível. No caso da restrição de capital, foi considerado, além do capital próprio, revelado pelas entrevistas, o financiamento para custeio, distinto entre grupos, previsto pelo PRONAF.

\section{RESULTADOS E DISCUSSÃO}

\section{Resultado da simulação do PRONAF A}

No trabalho foram considerados apenas financiamentos de custeio, ficando o Grupo A somente com a disponibilidade de capital próprio ( $\mathrm{R} \$ 5.500,00 / \mathrm{ano})$. Houve a limitação de área ( 3 ha) para todas as culturas, forçando a diversificação de atividades na resposta encontrada pelo modelo de programação linear. Seria arriscado o pequeno produtor investir em grandes áreas devido às condições limitadas de recursos disponíveis encontradas em seu ambiente rural. Sendo assim, a resposta encontrada para este Grupo A foi o investimento nas culturas da goiabeira, do maracujazeiro e pinheira ao longo dos dez anos (Tabela 2), obtendo o saldo final de R\$ 135.708,50.

Na solução ótima apresentada pelo programa, o valor do saldo final considera o pagamento de todos os custos operacionais efetivos das culturas, além do valor imputado à remuneração da mãode-obra familiar, de R \$ 5.520,00 por ano. Esse valor foi obtido multiplicando-se o número de trabalhadores equivalentes ao trabalho disponibilizado pelos membros da família, avaliado em 2,3 trabalhadores adultos, pelo salário mínimo ( $\$$ \$200,00), computado em doze meses. Os valores acima de zero no oitavo e nono anos de mão-deobra (MDO) apresentados na Tabela 2 significam que houve contratação de mão-de-obra na propriedade, pois a mão-de-obra familiar não foi suficiente neste período em virtude de maiores investimentos nas atividades escolhidas. 
TABELA 2 - Resultado da simulação do modelo de programação linear para o PRONAF A

\begin{tabular}{|c|c|c|c|c|c|c|c|c|c|c|c|c|c|c|}
\hline \multirow{2}{*}{ Ano } & \multicolumn{11}{|c|}{ Área a ser plantada (ha) } & \multirow{2}{*}{$\begin{array}{c}\text { Folga } \\
\text { (ha) }\end{array}$} & \multirow{2}{*}{$\begin{array}{l}\mathrm{MB} \\
(\mathrm{R} \$)\end{array}$} & \multirow{2}{*}{$\begin{array}{c}\text { MDO } \\
(\mathrm{d} / \mathrm{h})\end{array}$} \\
\hline & $1 *$ & 2 & 3 & 4 & 5 & 6 & 7 & 8 & 9 & 10 & $\Sigma$ & & & \\
\hline 1 & 0,00 & 0,00 & 0,00 & 0,00 & 0,00 & 0,39 & 0,31 & 0,00 & 0,00 & 1,45 & 2,15 & 7,85 & $11.279,04$ & 0,00 \\
\hline 2 & 0,00 & 0,00 & 0,00 & 0,00 & 0,00 & 1,85 & 0,31 & 0,00 & 0,00 & 1,00 & 3,16 & 6,84 & $9.666,71$ & 0,00 \\
\hline 3 & 0,00 & 0,00 & 0,00 & 0,00 & 0,00 & 1,85 & 0,31 & 0,00 & 0,00 & 1,19 & 3,35 & 6,65 & $13.310,58$ & 0,00 \\
\hline 4 & 0,00 & 0,00 & 0,00 & 0,00 & 0,00 & 3,00 & 0,31 & 1,10 & 0,00 & 0,37 & 4,78 & 5,22 & $13.362,47$ & 0,00 \\
\hline 5 & 0,00 & 0,00 & 0,00 & 0,00 & 0,00 & 3,00 & 0,43 & 3,01 & 0,00 & 0,00 & 6,44 & 3,56 & $12.382,19$ & 0,00 \\
\hline 6 & 0,00 & 0,00 & 0,00 & 0,00 & 0,00 & 3,00 & 0,43 & 3,01 & 0,00 & 0,29 & 6,73 & 3,27 & $28.454,47$ & 0,00 \\
\hline 7 & 0,00 & 0,00 & 0,00 & 0,00 & 0,00 & 3,00 & 1,28 & 3,01 & 0,00 & 0,00 & 7,29 & 2,71 & $35.866,64$ & 0,00 \\
\hline 8 & 0,00 & 0,00 & 0,00 & 0,00 & 0,00 & 3,00 & 2,90 & 3,01 & 0,00 & 0,00 & 8,91 & 1,09 & $41.001,25$ & 146,13 \\
\hline 9 & 0,00 & 0,00 & 0,00 & 0,00 & 0,00 & 3,00 & 2,90 & 3,01 & 0,00 & 0,00 & 8,91 & 1,09 & $52.176,00$ & 87,00 \\
\hline 10 & 0,00 & 0,00 & 0,00 & 0,00 & 0,00 & 3,00 & 1,74 & 3,01 & 0,00 & 0,03 & 7,78 & 2,22 & $45.895,64$ & 0,00 \\
\hline
\end{tabular}

$\mathrm{SF}(\mathrm{R} \$)=135.708,50$

*1=aipim (mandioca); 2=cana-de-açúcar; 3=bananeira; 4=coqueiro; 5=abacaxizeiro; 6=goiabeira; 7=maracujazeiro; 8=pinheira (fruta do conde); 9=citros; $10=$ Olerícolas; $\Sigma ?=$ somatório da área total de todas as culturas/ano; $\mathrm{SF}=$ saldo final.

TABELA 3 - Resultado da simulação do modelo de programação linear para o PRONAF C

\begin{tabular}{|c|c|c|c|c|c|c|c|c|c|c|c|c|c|c|}
\hline \multirow{2}{*}{ Ano } & \multicolumn{11}{|c|}{ Área a ser plantada (ha) } & \multirow{2}{*}{$\begin{array}{c}\text { Folga } \\
\text { (ha) }\end{array}$} & \multirow{2}{*}{$\begin{array}{l}\mathrm{MB} \\
(\mathrm{R} \$)\end{array}$} & \multirow{2}{*}{$\begin{array}{c}\mathrm{MDO} \\
(\mathrm{d} / \mathrm{h})\end{array}$} \\
\hline & $1 *$ & 2 & 3 & 4 & 5 & 6 & 7 & 8 & 9 & 10 & $\Sigma$ & & & \\
\hline 1 & 0,00 & 0,00 & 0,00 & 0,00 & 0,00 & 1,53 & 1,52 & 0,00 & 0,00 & 0,71 & 3,76 & 11,24 & $5.520,00$ & 0,00 \\
\hline 2 & 0,00 & 0,00 & 0,00 & 0,00 & 0,00 & 3,00 & 1,52 & 0,29 & 0,00 & 0,40 & 5,21 & 9,79 & $12.238,09$ & 0,00 \\
\hline 3 & 0,00 & 0,00 & 0,00 & 0,00 & 0,00 & 3,00 & 1,52 & 2,09 & 0,00 & 0,00 & 6,61 & 8,39 & $17.808,57$ & 0,00 \\
\hline 4 & 0,00 & 0,00 & 0,00 & 0,00 & 0,00 & 3,00 & 0,86 & 3,00 & 0,00 & 0,00 & 6,86 & 8,14 & $18.828,54$ & 0,00 \\
\hline 5 & 0,00 & 0,00 & 0,00 & 0,00 & 0,00 & 3,00 & 2,99 & 3,00 & 0,00 & 0,00 & 8,99 & 6,01 & $32.967,77$ & 159,62 \\
\hline 6 & 0,00 & 0,00 & 0,00 & 0,00 & 0,00 & 3,00 & 2,99 & 3,00 & 0,00 & 0,00 & 8,99 & 6,01 & $52.176,00$ & 87,00 \\
\hline 7 & 0,00 & 0,00 & 0,00 & 0,00 & 0,00 & 3,00 & 2,13 & 3,00 & 0,00 & 0,00 & 8,13 & 6,87 & $47.805,58$ & 17,86 \\
\hline 8 & 0,00 & 0,00 & 0,00 & 0,00 & 0,00 & 3,00 & 3,00 & 3,00 & 0,00 & 0,00 & 9,00 & 6,00 & $32.901,00$ & 189,00 \\
\hline 9 & 0,00 & 0,00 & 0,00 & 0,00 & 0,00 & 3,00 & 3,00 & 3,00 & 0,00 & 0,00 & 9,00 & 6,00 & $52.176,00$ & 87,00 \\
\hline 10 & 0,00 & 0,00 & 0,00 & 0,00 & 0,00 & 3,00 & 3,00 & 3,00 & 0,00 & 0,00 & 9,00 & 6,00 & $52.176,00$ & 87,86 \\
\hline
\end{tabular}

$\mathrm{SF}(\mathrm{R} \$)=183.000,40$

*1=aipim (mandioca); 2=cana-de-açúcar; 3=bananeira; 4=coqueiro; 5=abacaxizeiro; 6=goiabeira; 7=maracujazeiro; 8=pinheira (fruta do conde); 9=citros; $10=$ Olerícolas; $\Sigma ?=$ somatório da área total de todas as culturas/ano; $\mathrm{SF}=$ saldo final.

\section{Resultado da simulação do PRONAF C}

A resposta do modelo para o Grupo C foi o investimento nas culturas da goiabeira, do maracujazeiro e da pinheira, com o financiamento de custeio de $\mathrm{R} \$ 3.000,00$ a cada dois anos e limitação de área de 3 ha, sendo a mesma resposta do modelo do Grupo A. Entretanto, devido à maior disponibilidade de área (15 ha) e principalmente a de capital, houve maiores investimentos nestas culturas, acarretando em maiores contratações de mão-de-obra. No final dos dez anos o modelo obteve um saldo final de R $\$ 183.000,40$ (Tabela 3 ), maior do que o resultado do saldo final do modelo do Grupo A que não obteve financiamento de custeio. A contratação de mão-de-obra aconteceu a partir do quinto ano e a quantidade de área máxima otimizada aconteceu no oitavo, nono e décimo ano. Assim, relativamente ao Grupo A, a maior disponibilidade de recursos financeiros do Grupo $\mathrm{C}$ possibilitou maior aproveitamento da área e induziu à maior contratação de mão-de-obra, o que é importante indício das possibilidades de geração de empregos advindas do financiamento da agricultura familiar.

As margens brutas (MB) obtidas a cada ano foram incluídas no modelo de forma que a folga de cada ano, caso houvesse, fosse passada para o próximo ano de modo que o produtor aumentasse a sua quantidade de capital para investimento. De acordo com a Tabela 3, houve um aumento significativo das margens brutas a cada ano, propiciado pela concessão do crédito, o que evidencia a importância dos financiamentos concedidos pelo PRONAF no sentido de gerar maior renda e contribuir para a manutenção de condições mais favoráveis à permanência desses trabalhadores na atividade.

\section{Resultado da simulação do PRONAF D}

O resultado do modelo obtido para o Grupo $\mathrm{D}$, de acordo com a Tabela 4, com limitação das áreas das culturas em 3 ha, foi o mesmo obtido pelos Grupos A e C, ou seja, investimento ao longo dos anos nas culturas da goiabeira, do maracujazeiro e da pinheira. Contudo, pela maior capitalização dos produtores deste Grupo e maior disponibilidade de financiamento de custeio $(\mathrm{R} \$ 5.000,00)$, o saldo final $(\mathrm{SF}=\mathrm{R} \$ 187.710,30)$ foi o maior encontrado em relação a todos os grupos. Não houve investimento em novas culturas, mas sim uma redistribuição da área plantada entre as culturas de goiaba, maracujá e pinha, propiciando a obtenção de maior margem bruta. Constata-se ainda que houve uma maior folga de área durante os anos, ou seja, sobrou área de plantio não utilizada na simulação, evidenciando que uma maior quantidade de recursos financeiros poderia otimizar a disponibilidade de área do pequeno produtor.

Observa-se que o saldo final encontrado para o Grupo D não é muito superior ao saldo final do Grupo $\mathrm{C}$, podendo-se concluir que a maior quantidade de financiamento de custeio obtida pelo Grupo D não acarreta diferenças significativas em termos de escolha das atividades. Constatase assim que os agricultores do grupo D também têm suas opções econômicas limitadas pela escassez de recursos financeiros, ainda que, dentre todos os grupos, este seja o mais capitalizado. Portanto, devido à maior disponibilidade de área (20 ha) deste Grupo, quando comparado com os Grupos A (10 ha) e Grupo C (15 ha), sugere-se um aumento do financiamento de custeio pelo PRONAF, podendo o pequeno produtor melhorar a otimização de seus recursos e conseqüentemente aumentar sua renda. 
Tabela 4 - Resultado da simulação do modelo de programação linear para o PRONAF D

\begin{tabular}{|c|c|c|c|c|c|c|c|c|c|c|c|c|c|c|}
\hline \multirow{2}{*}{ Ano } & \multicolumn{11}{|c|}{ Área a ser plantada (ha) } & \multirow{2}{*}{$\begin{array}{c}\text { Folga } \\
\text { (ha) }\end{array}$} & \multirow{2}{*}{$\begin{array}{l}\text { MB } \\
(\mathrm{R} \$)\end{array}$} & \multirow{2}{*}{$\begin{array}{c}\text { MDO } \\
(\mathrm{d} / \mathrm{h})\end{array}$} \\
\hline & $1 *$ & 2 & 3 & 4 & 5 & 6 & 7 & 8 & 9 & 10 & $\Sigma$ & & & \\
\hline 1 & 0,00 & 0,00 & 0,00 & 0,00 & 0,00 & 1,30 & 1,82 & 0,00 & 0,00 & 0,71 & 3,83 & 16,17 & $5.520,00$ & 0,00 \\
\hline 2 & 0,00 & 0,00 & 0,00 & 0,00 & 0,00 & 3,00 & 1,82 & 1,19 & 0,00 & 0,00 & 6,01 & 13,99 & $10.928,00$ & 0,00 \\
\hline 3 & 0,00 & 0,00 & 0,00 & 0,00 & 0,00 & 3,00 & 1,82 & 2,39 & 0,00 & 0,00 & 7,21 & 12,79 & $18.126,88$ & 13,45 \\
\hline 4 & 0,00 & 0,00 & 0,00 & 0,00 & 0,00 & 3,00 & 1,57 & 3,00 & 0,00 & 0,00 & 7,57 & 12,43 & $23.474,63$ & 62,20 \\
\hline 5 & 0,00 & 0,00 & 0,00 & 0,00 & 0,00 & 3,00 & 3,00 & 3,00 & 0,00 & 0,00 & 9,00 & 11,00 & $39.299,53$ & 135,70 \\
\hline 6 & 0,00 & 0,00 & 0,00 & 0,00 & 0,00 & 3,00 & 3,00 & 3,00 & 0,00 & 0,00 & 9,00 & 11,00 & $52.176,00$ & 87,00 \\
\hline 7 & 0,00 & 0,00 & 0,00 & 0,00 & 0,00 & 3,00 & 1,77 & 3,00 & 0,00 & 0,00 & 7,77 & 12,23 & $43.787,10$ & 0,00 \\
\hline 8 & 0,00 & 0,00 & 0,00 & 0,00 & 0,00 & 3,00 & 3,00 & 3,00 & 0,00 & 0,00 & 9,00 & 11,00 & $35.066,15$ & 177,54 \\
\hline 9 & 0,00 & 0,00 & 0,00 & 0,00 & 0,00 & 3,00 & 3,00 & 3,00 & 0,00 & 0,00 & 9,00 & 11,00 & $52.176,00$ & 87,00 \\
\hline 10 & 0,00 & 0,00 & 0,00 & 0,00 & 0,00 & 3,00 & 2,66 & 3,00 & 0,00 & 0,00 & 8,66 & 11,34 & $50.471,85$ & 60,04 \\
\hline
\end{tabular}

$\mathrm{SF}(\mathrm{R} \$)=187.710,30$

*1=aipim (mandioca); $2=$ cana-de-açúcar; $3=$ bananeira; 4=coqueiro; 5=abacaxizeiro; $6=$ goiabeira; $7=$ maracujazeiro; $8=$ pinheira (fruta do conde); 9=citros; $10=$ Olerícolas; $\Sigma=$ somatório da área total de todas as culturas/ano; $\mathrm{SF}=$ saldo final.

\section{CONCLUSÕES}

1) Com base nos resultados desse trabalho, conclui-se que as variáveis tamanho da área e disponibilidade de mão-de-obra não foram limitantes na maximização do saldo final. Os recursos financeiros, por sua vez, foram identificados como a variável que mais limitação impõe à obtenção de maiores margens brutas pelos agricultores familiares. Esta limitação é menor para os agricultores pertencentes ao Grupo D, que, dentre os demais, se caracterizam por maior capitalização. Essa é a razão pela qual esse grupo obteve, na simulação feita, maior margem bruta e utilizou volume mais significativo de mão-de-obra. De todo o modo, pode-se concluir, a partir da análise feita, que a concessão de maiores volumes de financiamento para custeio propiciaria a esses agricultores, independentemente do grupo a que pertencem, melhor emprego da mãode-obra familiar e a geração de novos empregos no campo.

2) Os resultados possibilitaram concluir que as culturas do maracujazeiro, da goiabeira e da pinheira apresentam-se como atividade viável para o pequeno produtor da região Norte Fluminense, com grande potencial para a geração de renda e emprego. Embora tenha sido considerado apenas o financiamento de custeio, há outras linhas de financiamento no âmbito do PRONAF, com possíveis impactos sobre as condições de vida dos agricultores familiares, o que, entretanto, não foi considerado na análise feita. Finalmente, deve-se levar em conta as limitações dos dados e da metodologia empregada. Nesse sentido, é preciso considerar que, mais importantes do que os valores absolutos dos resultados obtidos, são as possibilidades advindas da concessão de crédito para os agricultores familiares, reproduzidas através da simulação feita.

3) Em função do potencial gerador de renda e emprego da fruticultura e da agroindústria processadora de frutas encontrada na região, é importante que sejam conduzidos novos estudos sobre a cadeia produtiva dessa atividade, visando conferir maior embasamento à escolha dessa opção como estratégia para o desenvolvimento econômico regional.

\section{REFERÊNCIAS BIBLIOGRÁFICAS}

BRASIL. Ministério do Desenvolvimento Agrário (2001). Programa Nacional de Fortalecimento da Agricultura Familiar-Pronaf. Disponível em $<$ http://www.pronaf.gov.br/saf/default.htm>, Acesso em 10 out. 2001.

FAO/INCRA. Perfil da agricultura familiar no Brasil: dossiê estatístico. Brasília, 1996.

FIBGE (2001) Censo Agropecuário1995/96. Disponível em: <http:// www.ibge.gov.br> Acesso em: 15/12/01 (página mantida pelo IBGE).

PRADO, D. Programação linear. Belo Horizonte: Editora de Desenvolvimento Gerencial, 1999, v. 2, 208p.

PUCCINI, A. D.; PIZZOLATO, N. D. Programação linear. Rio de Janeiro; São Paulo: LTC - Livros Técnicos e Científicos Editora, 1987. p.46-64. 